\title{
Reproducción en cautiverio de Crocodylus moreletii en Tabasco, México
}

\author{
Reproduction of Crocodylus moreletii in captivity in Tabasco, Mexico
}

\author{
Gustavo Casas-Andreu*, Gabriel Barrios-Quiroz y Rodrigo Macip-Ríos \\ Laboratorio de Herpetología, Departamento de Zoología, Instituto de Biología, Universidad Nacional Autónoma de México. Apartado postal 70-153, \\ Coyoacán 04510 México, D. F., México. \\ *Correspondencia: gcasas@ibiologia.unam.mx
}

\begin{abstract}
Resumen. Entre 1990 y 1993 se estudió la reproducción de Crocodylus moreletii en cautiverio, con 27 machos y 109 hembras. Las hembras resultaron en longitud hocico-cloaca (LHC) más grandes (60 a $140 \mathrm{~cm}$ ) que lo previamente conocido. Las hembras reproductivas más frecuentes presentaron tallas entre 81 y $100 \mathrm{~cm}$ de LHC. La longitud total mínima para la reproducción en hembras fue de $135 \mathrm{~cm}$. El cortejo, apareamiento, anidación, incubación y eclosión ocurrieron entre febrero y septiembre. El $8.25 \%$ de las hembras anidaron durante los 4 años de estudio, el $11 \%$ en 3 años, el $28.44 \%$ en 2 , y el $52.29 \%$ en un sólo año. Los eventos de cortejo y apareamiento mencionados se relacionaron con las temperaturas ambientales promedio más altas, y la anidación con el inicio de la temporada de lluvias. El número de huevos por nido varió entre 6 y 50 (29.24 \pm 8.72). La talla (LT y LHC) y la masa corporal son predictores regulares para las características reproductivas. No se encontró relación significativa entre las características del huevo y el tamaño de la nidada, siendo diferente a lo esperado. El tamaño y peso de las hembras no influyeron en el tamaño de la nidada, por lo que otros factores como los ambientales, la calidad reproductora de los machos, la condición física de las hembras, la carga de reproductores por estanque y la territorialidad pueden estar influyendo en este rasgo.
\end{abstract}

Palabras clave: Crocodylus moreletii, reproducción en cautiverio, fenología reproductora, anidación.

\begin{abstract}
Between 1990 and 1993 we studied the Morelet's crocodile reproduction in captivity, with a sample of 27 males and 109 females. Females were larger (60 to $140 \mathrm{~cm} \mathrm{SVL})$ than previously known. Most frequent size of nesting females was between 81 and $100 \mathrm{~cm}$ of SVL. Sexual maturity in females was attained at a minimum size of $135 \mathrm{~cm}$. The courtship, mating, nesting and hatching occurred between February and September. In the sample of females $8.25 \%$ nesting in each one of the 4 years of study, $11 \%$ in 3 years, $28.44 \%$ in 2, and $52.29 \%$ in one. The reproductive events mentioned were related to the highest average environmental temperatures, and nesting to the beginning of the rainy season. The number of eggs per nest fluctuated between 6 and 50 (29.24 \pm 8.72$)$. The body size and body mass were not good fine predictors of the reproductive characteristics. There was not correlation between egg characteristics and the clutch size, being different from the expected pattern. The body size and body mass were only slight correlated to clutch size, therefore, environmental factors, the reproductive quality of males, the physical condition of the females, the stocking rates, sex ratios, and territoriality may be influencing the reproductive traits.
\end{abstract}

Key words: Crocodylus moreletii, reproduction in captivity, reproductive phenology, nesting.

\section{Introducción}

Como respuesta a la disminución de las poblaciones de cocodrilos, en algunos países han surgido programas de conservación. Desde sus inicios los programas han variado considerablemente; sin embargo, la reproducción en cautiverio es un factor determinante, siendo la base para las estrategias de manejo y conservación de la mayoría de estos animales. Existen granjas o cultivos cerrados, conocidos como farming, donde se mantiene a los cocodrilos para la producción e incubación de huevos y eclosión

Recibido: 08 agosto 2009; aceptado: 11 enero 2010 de las crías, las cuales permanecen ahí hasta alcanzar tallas comerciales para obtención de productos derivados (Pooley, 1971; Joanen y McNease, 1987). En otro sistema denominado rancheo, ranching, los huevos y crías se obtienen, en estado silvestre y son llevados a cautiverio para su aprovechamiento una vez que tienen el tamaño requerido o para liberarlas en áreas naturales (Chabreck, 1967; Yadav, 1969; Honegger, 1971; Youngprapakorn, 1972; Bustard, 1974).

Pocos son los estudios de conservación de los cocodrilos y muchos los aspectos de su ecología que se desconocen; no obstante, en años recientes se han realizado investigaciones que revelan que mientras algunas poblaciones se mantienen en buen estado otras han sido 
extirpadas de sus hábitats (Campbell, 1972a; Abercrombie et al. 1980; Platt, 1996).

Crocodylus moreletii (cocodrilo de pantano) se encuentra incluido en el Apéndice II con cuota cero para ejemplares silvestres del Convenio sobre el Comercio Internacional de Especies Amenazadas de Flora y Fauna Silvestres (CITES). En el país está considerada como especie con protección especial (Pr) por la NOM-059ECOL-2010. De los cocodrilos de México, C. moreletii es el que mejores resultados ha mostrado para su cultivo (Casas-Andreu, 1995).

Martín de Lucenay (1942) realizó las primeras observaciones sobre reproducción y cultivo en cautiverio de $C$. moreletii en Veracruz, aunque sus resultados no tuvieron impacto en esos años. La anidación de esta especie en cautiverio la registró por primera vez Hunt $(1973,1975)$; además, realizó observaciones sobre el cuidado parental en cautiverio. Campbell (1972b) comparó la construcción del nido en forma de montículo que presenta esta especie, con la forma de anidación de otros cocodrilos, y realizó anotaciones sobre el significado filogenético del comportamiento. Casas-Andreu y Guzmán (1970) mencionaron que la temporada de mayor actividad para la reproducción de esta especie corresponde a los meses más cálidos del año (marzo a agosto). Estos mismos autores aportaron datos relacionados con la descripción de la especie y sus hábitats naturales, así como de la disminución en la distribución dentro del territorio nacional. Asimismo, Álvarez del Toro (1974) dio a conocer datos generales sobre la reproducción de C. moreletii. Pérez-Higareda (1980) realizó estudios sobre anidación del cocodrilo de pantano en el sur de Veracruz. El mismo autor y colaboradores publicaron algunas notas sobre el cortejo, conducta de apareamiento y sus hábitos alimentarios (Pérez-Higareda, et al., 1989). Los primeros datos cuantitativos que se conocen sobre la anidación de C. moreletii fueron publicados por Casas-Andreu y Rogel-Bahena (1986), y en el mismo año Huerta (1986) presentó la tesis en la que analizó diferentes parámetros de la especie y algunas características sobre su anidación, aunque infortunadamente no se publicó. Casas-Andreu et al. (1993) describieron algunos parámetros de la anidación de C. moreletii en cautiverio, y posteriormente, CasasAndreu y Barrios Quiroz (1997) generaron observaciones adicionales sobre la reproducción de esta misma especie.

Recientemente, Platt et al. (2008) describieron la ecología reproductiva y de anidación de C. moreletii en condiciones naturales en el norte de Belice entre los años de 1992 y 1995, donde encontraron que la masa de huevos se relaciona positivamente con la longitud hocico cloaca de la hembra y se incrementa al aumentar el tamaño de la puesta. Thorbjarnarson (1996) realizó un estudio donde definió la frecuencia reproductora, como el porcentaje de hembras adultas que anidan al año dentro de una población.

En vista de lo fragmentario de la información publicada sobre la reproducción de $C$. moreletii en cautiverio en México, y ante la necesidad de contar con suficiente información sobre la misma, durante los años de 1990 a 1993 se registraron anualmente datos sobre la reproducción de la especie. Los resultados preliminares de estas prácticas fueron publicados por Casas-Andreu et al. (1993) y CasasAndreu y Barrios-Quiroz (1997).

Dado que en adición a la previamente publicada se contó con más información al respecto, se decidió analizarla y comparar los resultados obtenidos con los registrados por Huerta (1986) y Platt et al. (2008) en condiciones naturales. Los datos generados en este trabajo serán de gran utilidad para valorar los programas de crianza en cautiverio, conservación y reintroducción de la especie, pues permitirán cotejar la limitada información existente en organismos silvestres con la establecida para las condiciones controladas de granja.

\section{Materiales y métodos}

Descripción del área de estudio. La granja de cocodrilos donde se desarrolló el estudio cuenta con una extensión de $7000 \mathrm{~m}^{2}$ aproximadamente; se encuentra ubicada en la ranchería Buenavista, primera sección, municipio del Centro, a $34 \mathrm{~km}$ al noreste de la ciudad de Villahermosa, Tabasco (18॰ 08' 28.7' N y 92 44'59.54" O; Fig. 1).

Según García de Miranda (1981), el clima es del tipo Am(f), que se define como cálido húmedo, caracterizándose por temperaturas elevadas, con un promedio anual mayor a los $26{ }^{\circ} \mathrm{C}$. La temperatura máxima promedio anual es de $32{ }^{\circ} \mathrm{C}$ y se presenta antes del inicio de la temporada de lluvias y del solsticio de verano. En tanto que la temperatura mínima promedio anual es de $20^{\circ} \mathrm{C}$ y se presenta en el mes de enero durante la temporada de nortes. La precipitación media mensual durante la temporada de lluvias es de 1700 a $2200 \mathrm{~mm}$, presentando una humedad relativa promedio del $75 \%$ (Fig. 2).

Colecta de datos. Entre los años de 1990 a 1993 se registraron los eventos preanidatorios (cortejo y apareamiento) y anidatorios (colecta, incubación y eclosión) de $C$. moreletii. Para ello, se contó con un lote de adultos de 19 machos y 51 hembras en 1990; 23 machos y 52 hembras en 1991; 22 machos y 47 hembras en 1992 y 24 machos y 41 hembras en 1993. En total fueron 109 las hembras con actividad reproductora. Aún cuando la frecuencia reproductora en cautiverio (porcentaje de hembras anidadoras por año) puede estar sujeta a diversas variables (debido al manejo de los animales), se consideró importante para 


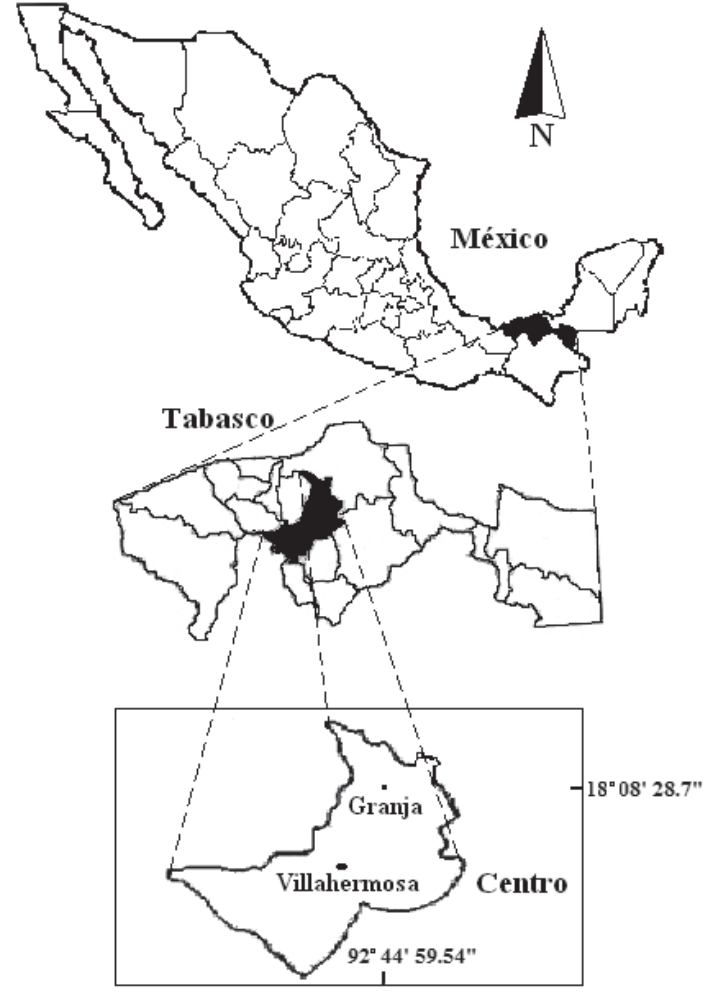

Figura 1. Localización de la granja de cocodrilos en el Municipio del Centro, Ejido Buenavista, 1ra. Sección, Tabasco, México.

el cultivo conocer la frecuencia de la anidación de las hembras dentro de la granja, a las cuales se les realizó un seguimiento durante la temporada de estudio, ya que muchas se reprodujeron durante los 4 años y otras dejaron de hacerlo en años alternos, así como la incorporación de nuevas hembras.

Se distribuyeron en 30 acuaterrarios machos y hembras de diferentes medidas y en diferente número; la proporción de sexos varió desde 1:1 hasta 1:7 macho/hembras. Todos los cocodrilos se identificaron individualmente con marcas de plástico de color amarillo, colocadas en la cola, con un número de identificación visible a distancia; además, se utilizó la técnica de corte de crestas de escamas caudales (Bolton, 1994). Los cocodrilos se pesaron con una báscula de $250 \mathrm{~kg}$ (precisión $10 \mathrm{~g}$ ) y medidos con un flexómetro (precisión $1 \mathrm{~mm}$ ) registrando la longitud total (LT) y longitud hocico-cloaca (LHC) antes de la temporada de reproducción.

Para describir el patrón de anidación y determinar la existencia de una correlación con la temperatura y la precipitación de la zona se utilizaron los promedios mensuales de los datos climáticos de la Estación Meteorológica de Villahermosa (a $30 \mathrm{~km}$ de la granja, Fig. 2).

Con la hembra más pequeña en reproducción, se deter- minó la talla (longitud total) a la que C. moreletii alcanza la madurez sexual; al mismo tiempo se estableció el mes de inicio del cortejo y apareamiento y se anotaron aspectos del comportamiento reproductivo.

La actividad de anidación se inició con el suministro de material vegetal dentro de cada estanque, el cual consistió en hojarasca de plátano o banano Musa paradisiaca y una mezcla de popal (Thania geniculata, Heliconia latispatha y Cyperus articulatus). La recolecta de los huevos se efectuó durante las 12 horas posteriores a la puesta, principalmente por la mañana o en las últimas horas de la tarde. Se evitó colocarlos sobre el suelo caliente o exponerlos directamente al sol para evitar la muerte de los embriones o causar nacimientos prematuros (Pooley, 1990). Posteriormente, se colocaron en contenedores de plástico con el sustrato suministrado o vermiculita (silicato de magnesio) y trasladados a la sala de incubación. La fertilidad se determinó por el desarrollo de una banda en la parte media del cascarón, dentro de las primeras 24 horas posteriores a la oviposición (Ferguson, 1985).

Se estableció el tamaño de la nidada al contar el número de huevos puestos por las hembras en cada uno de los 109 nidos colectados. Los huevos se midieron $(1 \mathrm{~mm})$ en largo (LH), ancho (AH), pesaron (1 g) y examinaron antes de colocarlos en los contenedores de plástico. Se desecharon los que presentaban perforaciones o fracturas y los infértiles, para evitar la proliferación de hormigas u otros insectos, así como para prevenir la contaminación bacterial de otros huevos, especialmente si los fluidos caen sobre estos (Hunt, 1980). La temperatura en el cuarto de incubación osciló entre los 29 y $34{ }^{\circ} \mathrm{C}$, con $85 \%$.de humedad en cada nido. Trascurridos 2 meses de incubación se revisaron los nidos para observar si habían ocurrido eclosiones. En caso positivo, las crías fueron marcadas inmediatamente (corte de escamas caudales), pesadas $(1 \mathrm{~g})$ y medidas (1 $\mathrm{mm})$. Posteriormente, se enjuagaron con agua corriente para eliminar cualquier residuo que pudiera infectarlas. Para aquellas que no eclosionaron durante este periodo se estableció un tiempo de espera de 4 a 5 días, y cuando no pudieron hacerlo por sí solas, debido a su posición dentro del huevo o a que la membrana testácea estuviera más dura de lo normal, se procedió a romper manualmente el cascarón para ayudarles a salir. Esta intervención se realizó para determinar el éxito de anidación (número de crías eclosionadas por nido). En algunos casos hubo nacimientos prematuros ocasionados por el sonido que producen las crías que nacen primero (Foggin, 1987).

Análisis estadísticos. Se realizaron análisis de regresión lineal simple entre tamaño del cuerpo (LT) y tamaño de nidada (TN) por año, así como entre el TN, peso de nidada (PN) y masa relativa de nidada (MRN) y con las características merísticas del huevo como el largo (LH), el ancho 
(AH) y el peso del huevo (PH) con los datos totales de los 4 años o los años disponibles. Se realizó un análisis de correlación de Pearson entre el peso de los huevos y el peso de las crías para determinar una posible correlación entre los rasgos. También, se aplicó una prueba de Kruskall-Wallis para determinar la existencia de diferencias significativas en el tamaño de la nidada por años. Todas las pruebas se realizaron con ayuda del programa estadístico JMP 5.0.1. y con un nivel de significancia del $\alpha=0.05$.

\section{Resultados}

Con el fin de presentar los resultados (fenología reproductora) en una secuencia lógica, se abordan primero los eventos preanidatorios (cortejo, apareamiento), que son aquellos que se llevan a cabo previamente a la puesta de huevos y en seguida los eventos anidatorios (anidación, incubación y eclosión), analizando a la vez la talla de las hembras y la frecuencia reproductora.

Eventos preanidatorios. El cortejo (definido por el comportamiento y la mayor actividad de los cocodrilos en este rubro) se inició a mediados del mes de febrero con temperaturas promedio del aire de 24 y $25^{\circ} \mathrm{C}$, cuando la temporada de nortes está concluyendo; llegó a su máxima actividad en abril, con una temperatura promedio de $28.9^{\circ} \mathrm{C}$, y terminó hacia fines de mayo, al principio de la temporada de lluvias (Fig. 2).

El apareamiento ocurrió desde finales de febrero hasta mediados de junio. Las temperaturas mínimas y máximas promedio para esta actividad durante la temporada de estudio oscilaron entre los 22.6 y los $33.7^{\circ} \mathrm{C}$ respectivamente. El comportamiento del apareamiento se determinó por la presencia de "abrazos" de los machos a las hembras y la cópula. El macho mete la cola debajo de la hembra y se ladea un poco, a veces la hembra se ladea también ligeramente y en esa posición se realiza el coito; el proceso tiene una duración aproximada de 15 minutos. Una pareja puede copular varias veces en la misma temporada y un macho puede aparearse con varias hembras. En el cortejo el macho emite fuertes rugidos que pueden escucharse a gran distancia y que tienen el propósito de llamado o reclamo sexual. En el seguimiento que se realizó a 40 hembras, el tiempo trascurrido entre el cortejo y la oviposición fue de $51.02 \pm 21.42$ días (23-106 días).

Eventos anidatorios. La talla mínima registrada en la madurez sexual de una hembra reproductora de Crocodylus moreletii en condiciones de cautiverio fue de $67 \mathrm{~cm}$ LHC y $135 \mathrm{~cm}$ LT, desconociéndose la edad.

La longitud total y peso de los machos (27) y hembras (109) sexualmente activos variaron considerablemente durante los 4 años de estudio (Cuadro 1).

De los 109 nidos observados, 107 (98.16 \%) fueron construidos por acumulación de material vegetal. El año de 1993 se observó que 2 hembras (1.84 \%) excavaron un

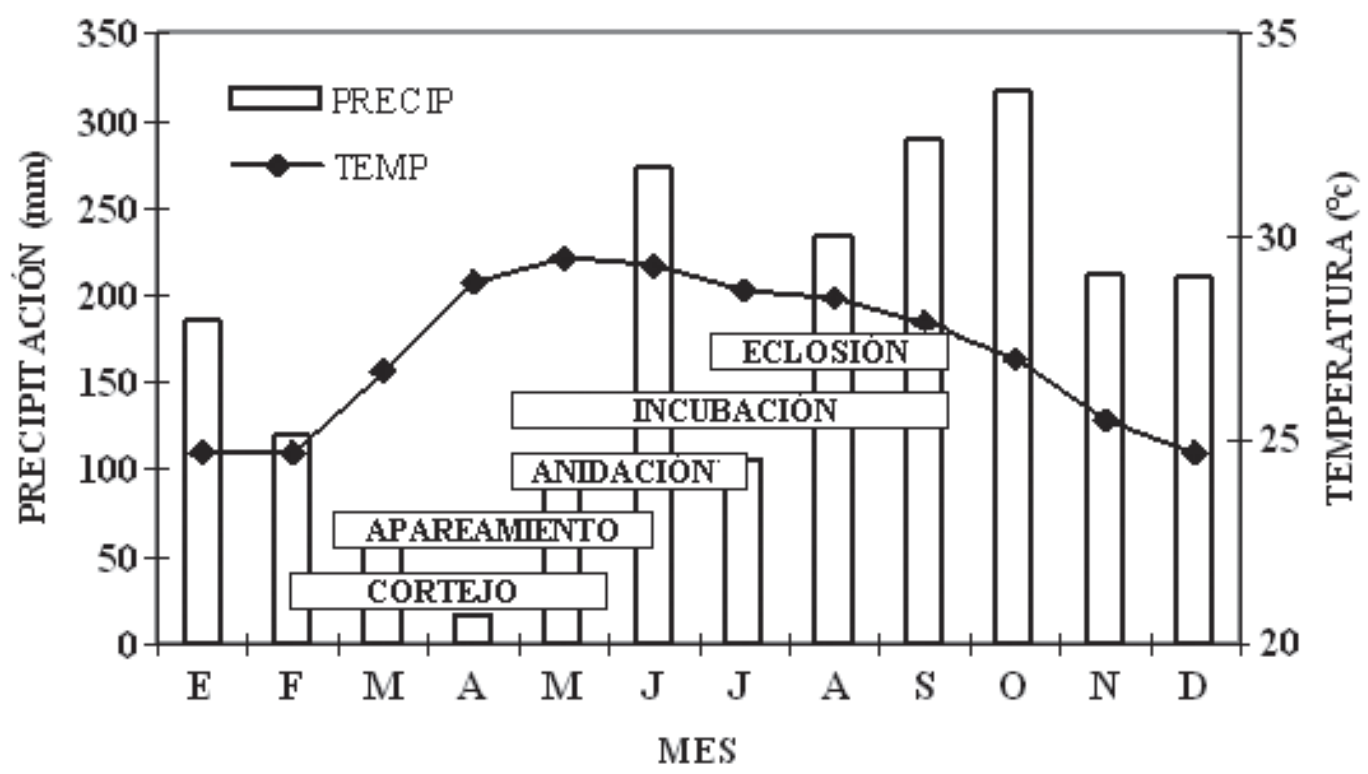

Figura 2. Climograma y fenología reproductora (cajas horizontales) de la granja de cocodrilos durante la temporada de estudio. Los datos climáticos fueron obtenidos de la estación meteorológica de Villahermosa, Tabasco, México. 
Cuadro 1. Intervalo de longitud total (LT) y peso de los organismos reproductores durante la temporada de estudio

\begin{tabular}{lcc}
\hline & Machos & Hembras \\
& & \\
\hline \multirow{2}{*}{ LT (m) } & $2.14-3.10$ & $1.35-2.53$ \\
& $(2.43 \pm 1.31)$ & $(1.89 \pm 0.18)$ \\
Peso $(\mathrm{kg})$ & $43.7-140$ & $10.3-80.2$ \\
& $(76.42 \pm 30.57)$ & $(35.8 \pm 11.8)$ \\
\hline
\end{tabular}

hoyo para desovar, al margen de que contaron con material vegetal suficiente para la construcción del nido.

Durante el estudio se observó que las hembras iniciaron la construcción del nido excavando el suelo y reuniendo hojarasca alrededor del sitio donde sería ubicado el montículo, hasta alcanzar una altura considerable para la puesta; también se observaron hembras que se encontraban posicionadas sobre su nido o vigilándolo desde un sitio cercano. El diámetro de 43 nidos revisados durante 1991 fue aproximadamente de 2 metros ( 1 a $3.7 \mathrm{~m}$ de intervalo), con una altura de $50 \mathrm{~cm}$ en promedio.

La frecuencia reproductora se desarrolló de la siguiente manera; de las 109 hembras, $9(8.25 \%)$ se reprodujeron en las 4 temporadas; 12 (11.0\%) en 3 temporadas; 31 (28.44 $\%)$ en 2 temporadas y 57 hembras (52.29\%) sólo en 1 temporada.

La anidación se inició a fines de abril (27 y 29 de abril para 1990 y 1991) y principios de mayo (2 y 11 de mayo para los años 1992 y 1993) (Cuadro 2). Durante el estudio se observó que una hembra (número 21 ) ovipositó al día siguiente de la primera lluvia intensa de la temporada, marcando el inicio de la anidación durante los 4 años de estudio. En el año de 1991 se observaron 4 nidos con anidación múltiple, 1 nido con 3 hembras y 3 nidos con 2 hembras, mientras que en 1992 anidaron en el mismo nido
3 hembras. En todos los casos, sólo la hembra dominante respondió agresivamente a nuestra presencia.

La cámara de los huevos en 43 nidos revisados midió en promedio $355 \mathrm{~mm}$ de diámetro exterior, $198 \mathrm{~mm}$ de diámetro interior y $235 \mathrm{~mm}$ de profundidad. El pico de anidación (mayor número de nidos puestos en una quincena) coincidió con los meses con temperaturas promedio mensuales más altas $\left(29.36^{\circ} \mathrm{C}\right)$. En 1990 no se pudo determinar el número de nidos; en 1991 fueron 25 nidos durante la primera quincena de mayo (48\%); en 1992, 18 nidos (38.3\%) y en 1993, 15 nidos (36.6\%); correspondiendo los 2 últimos años a la primera quincena de junio. La anidación concluyó el 19 de junio en 1990, el 5 de julio en 1991, el 13 de julio en 1992 y el 14 de julio en 1993 (Cuadro 2).

El total de nidos registrados fue de 51 en 1990, 52 en 1991, 47 en 1992 y 41 en 1993, es decir, un promedio de 47.7 nidos por temporada. El tamaño de nidada (TN) varió entre 6 y 50 huevos en los 4 años de estudio, con un promedio de $29.98 \pm 7.97$ huevos para 1990, 27.26 \pm 9.06 para $1991,29.42 \pm 8.10$ para 1992 y $30.31 \pm 9.75$ para 1993 . No se observaron diferencias significativas en el numero de huevos entre los distintos años $\left(\mathrm{H}_{3,187}=3.48, \mathrm{P}=0.323\right)$, habiéndose obtenido un promedio general de $29.24 \pm 8.72$ huevos para los 4 años.

La incubación coincidió en todos los casos con los meses más cálidos del año. El promedio mínimo y máximo de temperatura ambiente para 1990 fue 23.5 y $36.3^{\circ} \mathrm{C}$; para $1991,23.0$ y $36.5^{\circ} \mathrm{C}$; para $1992,22.5$ y $33.8^{\circ} \mathrm{C}$, y para $1993,23.5$ y $33.4^{\circ} \mathrm{C}$. El periodo de incubación fluctuó entre 63 y 76 días para 1990, con un promedio de $69 \pm$ 2.63; entre 57 y 75 para 1991, con un promedio de 67.82 \pm 3.28 ; entre 63 y 81 para 1992, con un promedio de 73.42 \pm 3.10 , y entre 63 y 72 en 1993, con un promedio de $68 \pm$ 2.23. El promedio de incubación para las 4 temporadas de estudio fue de $69.56 \pm 2.81$ días. Del total de huevos incubados en los 4 años de estudio, el promedio de la viabilidad

Cuadro 2. Anidación, incubación y eclosión para las 4 temporadas de estudio en Crocodylus moreletii en Tabasco (* quincena)

\begin{tabular}{|c|c|c|c|c|}
\hline Año & 1990 & 1991 & 1992 & 1993 \\
\hline Anidación & 27 abril-19 junio & 29 abril-5 julio & 2 mayo-13 julio & 11 mayo-14 julio \\
\hline Pico de anidación & Sin datos & $\begin{array}{c}\text { *1ra. mayo (25 nidos) } \\
48 \%\end{array}$ & $\begin{array}{c}\text { *1ra. junio (18 nidos) } \\
383 \%\end{array}$ & $\begin{array}{c}* \text { 1ra. junio (15 nidos) } \\
36.6 \%\end{array}$ \\
\hline Periodo de incubación & 27 abril - 28 agosto & 29 abril - 31 agosto & 2 mayo - 21 septiembre & $\begin{array}{l}11 \text { mayo - } 20 \\
\text { septiembre }\end{array}$ \\
\hline Periodo de eclosión & $\begin{array}{c}9 \text { julio - } 28 \\
\text { agosto }\end{array}$ & 18 julio - 13 agosto & 14 julio - 21 septiembre & 27 julio - 20 septiembre \\
\hline
\end{tabular}


fue del $73.15 \%$, en tanto que el porcentaje de eclosión promedio representó el $40.74 \%$ (Cuadro 3).

En cuanto a la LT de las hembras vs TN, la relación varió durante los años de estudio, obteniéndose los siguientes resultados: para 1990 una relación débil pero significativa $\left(\mathrm{r}^{2}=0.11, \mathrm{P}=0.0141, \mathrm{~N}=51\right)$; para 1991 una relación igualmente débil y significativa $\left(\mathrm{r}^{2}=0.14, \mathrm{P}=\right.$ $0.0059, \mathrm{~N}=52)$; en 1992 no se encontró relación $\left(\mathrm{r}^{2}=0.06\right.$, $\mathrm{P}=0.08, \mathrm{~N}=47)$ y finalmente, para 1993 se encontró una relación moderada y significativa $\left(\mathrm{r}^{2}=0.24, \mathrm{P}=0.001, \mathrm{~N}=\right.$ 41). La relación obtenida para todos los datos de las 4 temporadas de estudio fue débil pero significativa $\left(\mathrm{r}^{2}=0.11\right.$, $\mathrm{P}=0.01)$. Los resultados obtenidos al relacionar el peso de las hembras (M) vs TN, fueron similares a las anteriores obteniéndose los siguientes valores: para $1990\left(\mathrm{r}^{2}=0.14\right.$, $\mathrm{P}=0.005)$ y para $1993\left(\mathrm{r}^{2}=0.15, \mathrm{P}=0.009\right)$ la relación fue débil y significativa, mientras que para $1991\left(\mathrm{r}^{2}=0.06, \mathrm{P}=\right.$ $0.07)$ y $1992\left(\mathrm{r}^{2}=0.03, \mathrm{P}=0.18\right)$ no se encontró ninguna relación. La relación de M contra el peso de nidada (PN) sólo se realizó para el año de 1991, siendo el resultado significativo $\left(\mathrm{r}^{2}=0.13, \mathrm{P}=0.0074, \mathrm{~N}=52\right)$ (Cuadro 4).

El TN presentó una relación positiva y significativa con el PN $\left(\mathrm{r}^{2}=0.92, \mathrm{P}=0.0001\right)$, así como con la masa relativa de nidada $(\mathrm{MRN})\left(\mathrm{r}^{2}=0.58, \mathrm{P}=0.0001\right)$; sin embargo, no se relacionó con las características del huevo (largo, ancho y peso del huevo). El PN presentó una relación positiva y significativa aunque moderada con el ancho $(\mathrm{AH})$ $\left(r^{2}=0.21, \mathrm{P}=0.0006\right)$ y peso del huevo $(\mathrm{PH})\left(\mathrm{r}^{2}=0.19, \mathrm{P}=\right.$
0.001). Finalmente, la MRN no presentó ninguna relación con las características del huevo (Cuadro 5). De una muestra de 1400 huevos, el diámetro mayor fluctuó entre de 58 y $71 \mathrm{~mm}$, con un diámetro menor entre 34 y $42 \mathrm{~mm}$; el peso también tuvo variaciones entre 49.9 y 74.9 gramos.

Las crías de $C$. moreletii presentaron al nacer una longitud total promedio de $262 \pm 17 \mathrm{~mm}$ (intervalo de talla entre $235-282 \mathrm{~mm}$ ) de longitud total. En tanto que para el peso la variación fue considerable, 34.9 a $55.5 \mathrm{~g}$, con un promedio de $45.1 \pm 1.17 \mathrm{~g}$, para los 4 años de estudio. El número de crías por nidada fue de $11.94 \pm 12$ con un intervalo de 1 a 40 crías. El peso promedio de los huevos y el peso promedio de las crías al nacer se correlacionaron de manera positiva y significativa $(r=0.72, \mathrm{p}<0.0001)$.

\section{Discusión}

En este apartado, se siguió la misma secuencia que en Resultados, es decir, primero los eventos preanidatorios seguidos de los anidatorios, la talla de las hembras y la frecuencia reproductora.

Eventos preanidatorios. El comportamiento de C. moreletii mostrado durante el cortejo fue similar al descrito previamente por otros autores (Garrik y Lang, 1977; Huerta, 1986).

Platt et al. (2008) mencionaron que el cortejo de $C$. moreletii en Belice tuvo lugar a fines de la época de secas

Cuadro 3. Eventos reproductores de Crocodylus moreletii en Tabasco (1990-1993)

\begin{tabular}{|c|c|c|c|c|}
\hline Parámetros & 1990 & 1991 & 1992 & 1993 \\
\hline Núm.. de nidos & 51 & 52 & 47 & 41 \\
\hline Huevos incubados & Sin datos & 1418 & 1383 & 1243 \\
\hline Incubación (días) & $\begin{array}{c}63-73 \\
(69 \pm 2.63)\end{array}$ & $\begin{array}{c}57-75 \\
(67.82 \pm 3.28)\end{array}$ & $\begin{array}{c}63-81 \\
(73.42 \pm 3.10)\end{array}$ & $\begin{array}{c}63-72 \\
(68 \pm 2.23)\end{array}$ \\
\hline Huevos por nido & $29.98 \pm 7.97$ & $27.26 \pm 9.06$ & $29.42 \pm 8.10$ & $30.31 \pm 9.75$ \\
\hline Crías por nido & $8.43 \pm 10.3$ & $9.46 \pm 10.12$ & $15.31 \pm 13.01$ & $14.58 \pm 12.29$ \\
\hline$\%$ viabilidad & Sin datos & 76.58 & 73.53 & 69.34 \\
\hline$\%$ inviabilidad & Sin datos & 23.41 & 26.47 & 30.66 \\
\hline$\%$ eclosiones & 28.12 & 34.69 & 52.06 & 48.1 \\
\hline
\end{tabular}


Cuadro 4. Análisis de tamaño corporal y peso vs valores reproductores en Crocodylus moreletii en la granja Buenavista

\begin{tabular}{|c|c|c|c|c|c|c|}
\hline & $T N$ & $L H$ & $A H$ & $P H$ & $P N$ & $M R N$ \\
\hline LHC & $\begin{array}{c}\mathrm{r}^{2}=0.061 \\
\mathrm{P}=0.07\end{array}$ & $\begin{aligned} \mathrm{r}^{2} & =0.151, \\
\mathrm{P} & =0.004\end{aligned}$ & $\begin{array}{l}\mathrm{r}^{2}=0.232 \\
\mathrm{P}=0.0003\end{array}$ & $\begin{array}{l}\mathrm{r}^{2}=0.278 \\
\mathrm{P}<0.0001\end{array}$ & $\mathrm{r}^{2}=0.12, \mathrm{p}=0.01$ & $\begin{array}{c}\mathrm{r}^{2}=-0.063 \\
\mathrm{P}=0.07\end{array}$ \\
\hline LT & $\begin{array}{l}\mathrm{r}^{2}=0.112 \\
\mathrm{P}=0.0141\end{array}$ & $\begin{aligned} \mathrm{r}^{2} & =0.133 \\
\mathrm{P} & =0.007\end{aligned}$ & $\begin{array}{l}\mathrm{r}^{2}=0.245 \\
\mathrm{P}=0.0002\end{array}$ & $\begin{array}{l}\mathrm{r}^{2}=0.230 \\
\mathrm{P}=0.0003\end{array}$ & $\begin{array}{l}\mathrm{r}^{2}=0.179 \\
\mathrm{P}=0.0018\end{array}$ & $\begin{aligned} \mathrm{r}^{2} & =-0.058 \\
\mathrm{P} & =0.085\end{aligned}$ \\
\hline $\mathrm{M}$ & $\begin{array}{c}\mathrm{r}^{2}=0.055 \\
\mathrm{P}=0.09\end{array}$ & $\begin{aligned} \mathrm{r}^{2} & =0.196 \\
\mathrm{P} & =0.001\end{aligned}$ & $\begin{array}{l}\mathrm{r}^{2}=0.233 \\
\mathrm{P}=0.0003\end{array}$ & $\begin{array}{l}\mathrm{r}^{2}=0.322 \\
\mathrm{P}<0.0001\end{array}$ & $\begin{array}{l}\mathrm{r}^{2}=0.135 \\
\mathrm{p}=0.0074\end{array}$ & $\begin{array}{l}\mathrm{r}^{2}=-0.136 \\
\mathrm{P}=0.0071,\end{array}$ \\
\hline
\end{tabular}

LHC, longitud hocico cloaca; TN, tamaño de la nidada; LT, longitud total; LH, largo del huevo; AH, ancho del huevo; PH, peso huevo; PN, peso de nidada; MRN, masa relativa de la nidada; M, masa corporal. Un guión, - , indica que hay una relación del tipo negativo o inversa. En las celdas sin signo, la relación o es positiva (cuando es significativa) o no existe relación aparente.

Cuadro 5. Análisis de regresión lineal entre rasgos reproductores de la población de Crocodylus moreletii en la granja Buenavista

\begin{tabular}{|c|c|c|c|c|c|}
\hline & $L H$ & $A H$ & $P H$ & $P N$ & $M R N$ \\
\hline $\mathrm{TN}$ & $\mathrm{r}^{2}=0.001, \mathrm{P}=0.81$ & $\mathrm{r}^{2}=0.082, \mathrm{P}=0.03$ & $\mathrm{r}^{2}=0.038, \mathrm{P}=0.165$ & $\mathrm{r}^{2}=0.92, \mathrm{P}<0.0001$ & $\mathrm{r}^{2}=0.58, \mathrm{P}<0.0001$ \\
\hline $\mathrm{PN}$ & $\mathrm{r}^{2}=0.064, \mathrm{P}=0.06$ & $\mathrm{r}^{2}=0.211, \mathrm{P}=0.0006$ & $\mathrm{r}^{2}=0.194, \mathrm{P}=0.0011$ & Sin datos & $\mathrm{r}^{2}=0.48, \mathrm{P}<0.0001$ \\
\hline MRN & $\mathrm{r}^{2}=0.004, \mathrm{P}=0.65$ & $\mathrm{r}^{2}=0.001, \mathrm{P}=0.777$ & $\mathrm{r}^{2}=0.0002, \mathrm{P}=0.92$ & Sin datos & Sin datos \\
\hline
\end{tabular}

TN, tamaño de la nidada; LH, largo del huevo; AH, ancho del huevo; PH, peso huevo; PN, peso de nidada; MRN, masa relativa de la nidada. Todas las relaciones son positivas, o bien, no existe relación aparente.

entre abril y mayo. En la granja el cortejo se inició a mediados de febrero, con un máximo en abril y concluyendo en mayo, es decir, ocurrió antes que lo descrito por Platt et al. (2008). El tiempo trascurrido entre el cortejo y la oviposición coincidió con el estudio de Huerta (1986), quien obtuvo datos similares para la misma especie en Ciudad del Carmen, Campeche, a temperaturas promedio mínimas y máximas de 16 y $36^{\circ} \mathrm{C}$, respectivamente. De igual forma, Álvarez del Toro (1974) señaló que del apareamiento a la postura transcurren de 1 mes y medio a 2 meses. PérezHigareda et al. (1989) registraron que el apareamiento en el sur de Veracruz se inicia a mediados de marzo, con un máximo de actividad en abril, a temperaturas que oscilan entre 28 y $31^{\circ} \mathrm{C}$. Lo que se puede determinar con base en los registros anteriores es que el apareamiento puede variar de un lugar a otro de acuerdo con las condiciones locales. Es posible que en los lugares con mayor efecto de frentes fríos del norte sea más tardía. Considerando el trabajo de Pérez-Higareda et al. (1989) la reproducción es temprana; el área de trabajo se encuentra latitudinalmente en la distribución sur de C. moreletii, este patrón es simi- lar al señalado por Casas (2003) para C. acutus, aunque desconocemos si estas condiciones son significativas en la estrategia reproductiva de la especie. Ferguson (1985), Lance (1987) y Joanen y McNease (1989) indicaron que durante el ciclo reproductor, la temperatura es un factor de gran importancia para la incubación de los huevos y determinación del sexo, y de manera particular muestran algunos datos para Alligator mississippiensis.

Eventos anidatorios. Los cocodrilos de este estudio corresponden a una especie de tamaño medio en comparación con otras especies (Greer, 1975; Casas-Andreu y Rogel, 1986). La longitud total (LT) de $135 \mathrm{~cm}$ de una hembra reproductora fue inferior a la que registran Huerta (1986), Platt (1996) y Platt et al. (2008), quienes señalan una talla mínima de150 cm LT para llegar a la reproducción. Éste es el primer registro para $C$. moreletii y se desconoce si se debió a factores como densidad, alimentación o temperatura.

Han sido registradas longitudes similares en otras especies; para Caiman crocodilus crocodilus se menciona una hembra reproductora con $1.30 \mathrm{~m}$ de LT, y menores, como 
en Caiman crocodilus fuscus, con $1.08 \mathrm{~m}$ de LT, aunque están consideradas como especies más pequeñas (Staton y Dixon, 1977). También se menciona que la madurez sexual está relacionada con el tamaño de la hembra, la cual se encuentra condicionada por factores como la temperatura y el alimento disponible (Staton y Dixon, 1977).

La longitud hocico cloaca de las hembras anidadoras presentó un intervalo mayor que el encontrado por Platt et al. (2008), quienes citan valores entre 60 y $120 \mathrm{~cm}$, diferencia que puede deberse a que la muestra analizada en este estudio fue mayor a la manejada por Platt et al. (16 hembras). Un dato interesante es que tanto en la población analizada en este estudio (a pesar de estar en cautiverio) como en la de Belice (Platt et al. 2008), las tallas más frecuentes en la población reproductiva se encontraron entre 81 y $100 \mathrm{~cm}$ LHC, y que podrían ser las de hembras que dominan en la población, posiblemente por su experiencia, competitividad y su atracción para los machos. Esto se observó en los estanques con más de una hembra (Fig. 3).

Por lo que respecta a la frecuencia reproductora, una de las posibles causas en la variación es que por ser animales en cautiverio el manejo pueda tener un papel importante, ya que en el caso de 2 encierros, al cambiar los machos, las hembras comenzaron a reproducirse al año siguiente, lo que pudo afectar los resultados de este estudio. Lo anterior sugiere que la calidad reproductora del macho es también un factor importante. Otra de las causas puede ser que las hembras se estén incorporando por primera vez a la población reproductiva, como es el caso de 2 hembras anidadoras que se encontraban en un acuaterrario que hasta ese momento se había considerado como de hembras y machos preadultos ( 97 organismos).

Thorbjarnarson (1996) registró la frecuencia reproductora en 7 especies de cocodrilos y encontró que entre los aligatóridos es entre 25 y $54 \%$ (media $=40.9 \%$ ) y entre 63 y $90 \%$ (media $=78.3 \%$ ) para los crocodílidos, de lo que se deduce que en la granja de cocodrilos la frecuencia reproductora de las hembras se encuentra por debajo de los resultados obtenidos para otras especies de cocodrilos. Aunque no se han realizado estudios previos en esta especie, se estima que eso pudo ser resultado de que durante la temporada de estudio, la manutención de los adultos en la granja se basó en pulmón de res, alimento que no reúne los requisitos necesarios para un buen ciclo reproductor (De la Ossa Velásquez y Sampedro Marín, 2001). Otra de las causas pudo haber sido la falta de espacio para un adecuado desarrollo. Estos 2 factores son señalados por Bucio (1992) como determinantes para tener una reproducción y

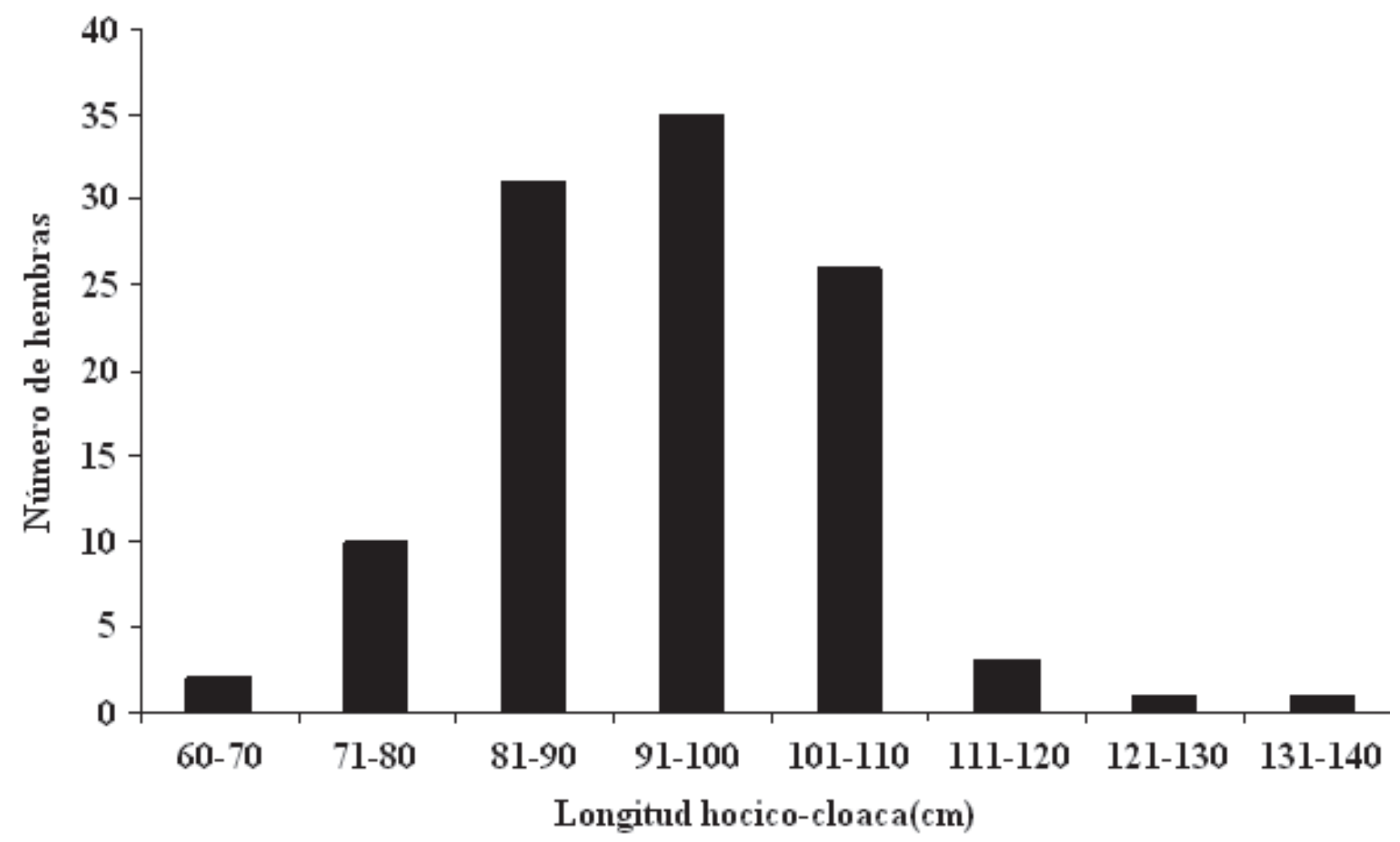

Figura 3. Clases de talla de hembras reproductoras de Crocodylus moreletii en la granja de Buenavista, Tabasco, México. 
crecimiento óptimos. De la misma manera, todos los factores mencionados pueden estar actuando sinérgicamente.

En este estudio se observó anidación múltiple durante los años de 1991 y 1992, siendo éste, después del trabajo realizado por Huerta (1986), el segundo registro de anidación colectiva para especies que anidan en montículo. Solamente se había registrado la anidación múltiple en especies que hacen nidos en hoyos (Lang, 1987). En el año de 1993, a pesar de tener material suficiente para la construcción del nido, 2 hembras hicieron un hoyo para desovar, y posteriormente en ese hoyo se encontraron huevos viables, con lo que podemos reafirmar, de acuerdo con lo descrito por Greer (1970), Campbell (1972b) y Brazaitis (1973), que en ocasiones C. moreletii también anida en hoyo. Platt et al. (2008) consideraron que probablemente la anidación en hoyos de C. moreletii en el norte de Belice sea una confusión de los investigadores por la morfología similar con Crocodylus acutus y, en términos generales, por la simpátria de estas especies en esa zona, sugiriendo que la anidación en hoyo por parte de C. moreletii puede ser el resultado de hibridización entre ambas especies. No obstante, es necesaria más investigación al respecto.

La puesta de huevos se inició al día siguiente de la primera lluvia fuerte de la temporada, factor que consideramos como detonante del inicio de la temporada de anidación, lo que concuerda con Huerta (1986) y Platt et al. (2008), quienes hicieron la misma observación. La anidación comenzó desde finales de abril (27-29), coincidiendo con el incremento de la temperatura promedio mensual del aire que se encontraban por arriba de $\operatorname{los} 29^{\circ} \mathrm{C}$ y con el inicio de la temporada de lluvias, tal como Huerta (1986) señala, quien propone que las lluvias y la temperatura son esenciales como factor desencadenante de la anidación, ya que estas condiciones son importantes para un buen desarrollo de los huevos y posterior eclosión de las crías. El pico de anidación para los años de estudio coincidió con los meses en los cuales la temperatura ambiental promedio fue mayor $\left(29.36^{\circ} \mathrm{C}\right)$. Este mismo comportamiento sucede en A. mississippiensis (Ferguson, 1985; Joanen y McNease, 1989).

La anidación en la granja concluyó a mediados de julio, correspondiendo con lo que previamente Casas-Andreu y Rogel (1986) indicaron como límite de anidación. Podemos resumir que la anidación en Tabasco se inicia a fines de abril o principios de mayo, pudiendo variar de un año a otro, y concluye hacia mediados de julio, ampliándose la temporada por casi un mes en este estudio, en relación con lo registrado por Platt et al. (2008) que la sitúan entre fines de mayo y mediados de junio, aun cuando la latitud de la localidad de la población de Belice es similar a la estudiada. Por ahora se desconoce el efecto de la latitud en la fenología reproductiva de C. moreletti, pero es posi- ble que cambie a lo largo de un gradiente latitudinal en respuesta a las condiciones del medio ambiente local, sin tener evidencias suficientes para señalar si estas condiciones son significativas en la estrategia reproductiva de la especie (Thorbjarnarson, 1989; Casas-Andreu, 2003). Sin embargo, se pudo observar que los factores climáticos y los rasgos de historia de vida (cortejo, apareamiento, anidación, incubación y eclosión) se encuentran muy relacionados con los aumentos de temperatura y precipitación (Fig. 2), en forma similar a lo encontrado por CasasAndreu y Rogel Bahena (1986) y Platt et al. (2008). Por su parte Webb y Cooper-Preston (1989) establecieron que la anidación en los cocodrilos se lleva a cabo cuando la temperatura y precipitación se sincronizan, aunque puede haber variaciones dependiendo de gradientes latitudinales y de las variaciones ambientales locales.

Los montículos de anidación de 43 nidos revisados durante el año de 1991 tuvieron en promedio un diámetro mayor que lo registrado por Platt et al. (2008). La cámara de los huevos en estos mismos nidos midió en promedio $355 \mathrm{~mm}$ de diámetro exterior y $198 \mathrm{~mm}$ de diámetro interior, con una profundidad promedio de $235 \mathrm{~mm}$, siendo más amplios que lo señalado por Platt et al. (2008). Lo anterior pudo deberse al tamaño de las hembras utilizadas en este estudio, de acuerdo con los datos anteriormente señalados, o a la cantidad del material suministrado para la construcción del nido.

Platt et al. (2008) encontraron en 73 nidos un tamaño de nidada variable, entre 9 y 42 huevos, con un promedio de $25 \pm$ 7.6. Huerta (1986) observó promedios de 29.8, $30.7,28.9$ y 36.3 , con un promedio general de 31.4 huevos para los 4 años de observaciones, mientras que CasasAndreu y Rogel (1986) determinaron un promedio de 30 huevos por nido. No obstante, en este estudio se encontró que el número de huevos puestos por nido varió entre 6 y 50 con un promedio de $29.24 \pm 8.72$. En este estudio se incrementa el intervalo máximo y mínimo de huevos por nido, mientras que el promedio es similar al de otros estudios con una tendencia promedio alrededor de los 30 huevos. Estos resultados denotan que la variación interpoblacional en el tamaño de nidada es reducida, lo cual no es raro, ya que las condiciones ambientales son similares en las diferentes zonas de estudio.

La relación entre el tamaño y peso de las hembras con el número de huevos presentó tendencias positivas y significativas en algunos años, pero el porcentaje de la varianza antes explicada es poco; sin embargo, se muestra una tendencia esperada como en otros vertebrados ectotermos (Charnov, 1993), (11 - $24 \%$ para LT), (13 - $32 \%$ peso), y es interesante que en otros años no exista relación alguna. A pesar de lo anterior, Álvarez del Toro (1974) y Platt et al. (2008) concluyeron que existe relación entre estos fac- 
tores. Al igual que Hall y Johnson (1987) en Crocodylus novaeguineae y Thorbjarnarson y Hernández (1993) para Crocodylus intermedius también indicaron una correlación entre el tamaño de la nidada $v s$ la longitud total de la hembra y la masa de la nidada. Por el contrario, en otras especies, tales como A. mississippiensis (Deitz y Hines, 1980; Joanen y McNease, 1987; Joanen y McNease, 1989), Crocodylus porosus (Webb et al., 1977), C. moreletii, (Casas-Andreu y Rogel, 1986), Caiman latirostris (Verdade, 2001; Larriera et al., 2004) no encontraron correlaciones significativas entre tamaño de nidada vs longitud total de la hembra. Es importante señalar que los estudios anteriores únicamente se realizaron en una sola temporada, lo que no ocurrió en este trabajo, y eso podría marcar la diferencia. Por consiguiente, el tamaño y peso del cuerpo no son componentes tan importantes en el tamaño de la nidada; existen otros factores que pueden estar influyendo, como los ambientales la calidad de machos reproductores seleccionados, la condición física (o de salud) de las hembras, el tamaño de cada estanque, el número de reproductores y, en este caso, la territorialidad, por las condiciones de cautiverio (Joanen y McNease (1989).

Casas-Andreu y Rogel (1986), en su análisis sobre nidadas de C. moreletii, señalan que existe una relación entre ancho y peso promedio de los huevos, correspondiendo el mayor peso a los más anchos; al correlacionar el peso de las hembras con el peso promedio de la nidada, el resultado fue significativo, además encuentran una relación entre el peso promedio de los huevos por nidada y el tamaño de la misma, teniendo las nidadas más numerosas los huevos más pesados. Esto confirma parte de los resultados obtenidos en este estudio.

En conclusión, en esta población de cocodrilos los datos de talla (LT y LHC) son predictores regulares de las características reproductivas, ya que, en algunos de los años muestreados los datos se comportan según lo esperado teóricamente y en otros no. A mayor masa corporal o talla, mayores valores en las variables reproductoras, con excepción del tamaño de nidada y la MRN (con una relación negativa). Lo anterior parece indicar que los individuos más grandes presentan valores más bajos de MRN en comparación con los más pequeños. Esto mismo se ha encontrado en otros reptiles como en algunas tortugas (Iverson et al. 1991, 1997)

Teóricamente, se esperaría encontrar trade-offs (indicados por relaciones negativas) entre los rasgos reproductores, como tamaño de la nidada $v s$ tamaño del huevo, ya que cuando aumenta el número de huevos, por lo general disminuye su tamaño, por restricciones energéticas y de espacio (Stearns, 1992); no obstante, esto no se encontró en ninguno de los casos, tal como lo documentaron Platt et al. (2008). La relación no significativa entre las carac- terísticas del huevo y el tamaño de la nidada es un dato diferente a lo esperado, ya que los trade-off típicos indican que a mayor número de huevos, menor tamaño de éstos. Quizá el dato más interesante es la estrecha relación entre el tamaño de nidada y el peso de la nidada, ya que se espera que mientras más huevos sean depositados, éstos tiendan a ser más pequeños y ligeros (Stearns, 1992); sin embargo, en este análisis se observa que las nidadas más grandes son las más pesadas cuando los datos se analizan en conjunto, es decir, no como en el caso del peso promedio del huevo.

El periodo de incubación para C. moreletii tiene menor duración que los registrados para otras especies de cocodrilos (Alcalá et al., 1987; Hall y Johnson, 1987; Thorbjarnarson y Hernández, 1993; Casas-Andreu, 2003). Consideramos que esto ocurre por las condiciones controladas que se mantuvieron durante la incubación y donde el intervalo de oscilación térmica y la humedad fue corto, lo cual contribuye a un mejor y más rápido desarrollo (Ferguson, 1985).

Por lo que respecta al número de crías por nido, la eclosión anual promedio en relación con los huevos viables durante el transcurso del estudio fue de $40.74 \%$. A pesar de que en la dieta de los reproductores no se suministró carne roja, la eclosión se encuentra cercana a los valores de más alta viabilidad (50\%) encontrados en A. mississipiensis. Particularmente, se ha registrado que las tasas de eclosión en A. mississippiensis cautivos, variaron entre 50 y $70 \%$ para reproductores alimentados con nutrias (carne roja), mientras que para los alimentados con pescado la tasa de eclosión fue de 23 a $61 \%$ con un promedio de 27.4 $\%$ (Joanen y McNease, 1987).

El promedio de longitud total al momento de nacer fue mayor al obtenido por Del Real (1983) y Huerta (1986), pero muy similar a lo obtenido por Platt et al. (2008) 269 $\pm 13 \mathrm{~mm}$, lo que podría ser el resultado de que las hembras utilizadas en este estudio y en el realizado por Platt et al. (2008) fueron de mayor talla que las utilizadas por los primeros autores, ya que se ha observado en otras especies de cocodrilos una relación de este tipo (Ferguson, 1985).

La correlación positiva y significativa entre el peso promedio de las crías y el peso promedio de los huevos podría indicar que con huevos más pesados se obtendrían crías más grandes, y posiblemente éstas podrían tener mayor tasa de sobrevivencia, aunque sería necesario tener datos de estos organismos en las etapas posteriores de su ciclo de vida para corroborar esta hipótesis. En otros cocodrilos, como Caiman c. crocodilus, A. mississippiensis, Caiman latirostris, también se ha encontrado esta correlación y como posibles causas se señalan el estado nutricional de la hembra al momento de la anidación, la edad, el tamaño, los factores ambientales (niveles de contaminantes) y adaptativos (actuando sobre ellas presiones selectivas que 
determinarían una mortalidad reducida en un periodo largo de sequía) (Staton y Dixon, 1977; Deitz y Hines, 1980; Larriera et al., 2004).

\section{Agradecimientos}

Los autores agradecen al Instituto de Biología de la Universidad Nacional Autónoma de México, al Dr. Andrés Resendiz Medina (q.e.p.d.), y a los biólogos José Francisco Iracheta y Horacio Saracho Vensamoye, de la entonces Secretaría de Desarrollo del Gobierno del Estado de Tabasco, por su apoyo logístico, sin el cual no se hubiera podido llevar a cabo esta investigación; al personal de la granja de cocodrilos, en especial al MVZ Armando de la Torre Espinoza, por su valioso apoyo en las labores de campo. Finalmente, a los revisores anónimos que con sus oportunos comentarios enriquecieron este trabajo.

\section{Literatura citada}

Abercrombie, C., L. D. Davidson, C. A. Hope y D. E. Scott. 1980. Status of Morelet's crocodile (Crocodylus moreletii) in Belize. Biological Conservation 17:103-113.

Alcalá, A. C., C. A. Ross y E. L. Alcalá. 1987. Observations on reproduction and behaviour of captive philippine crocodiles (Crocodylus mindorensis). Silliman Journal 34:18-28.

Álvarez del Toro, M. 1974. Los Crocodylia de México. Instituto Mexicano de Recursos Naturales Renovables. México, D. F. $70 \mathrm{p}$.

Bolton, M. 1994. La explotación del cocodrilo en cautividad. Guía FAO Conservación. 22:156 p.

Brazaitis, P. 1973. The identification of living crocodilians. Zoológica 58:59-88.

Bucio, G. A. 1992. Estudios para el diseño de un sistema de cría intensiva de cocodrilos (Crocodylus moreletii) jóvenes. Tesis de licenciatura, Facultad de Ciencias, Universidad Nacional Autónoma de México. México D. F. 44 p.

Bustard, H. R. 1974. Captive breeding of crocodile. Institute Rajendranagar, Hyderabad [India]. 20 p.

Campbell, H. W. 1972a. Preliminary report. Status investigation of Morelet's Crocodile in Mexico. Zoologica 57:135-136.

Campbell, H. W. 1972b. Ecological or phylogenetic interpretation of crocodilian nesting habits. Nature 238:404-405.

Casas-Andreu, G. 1995. Los cocodrilos de México como recurso natural, presente, pasado y futuro. Revista Sociedad Mexicana de Historia Natural 46:153-162.

Casas-Andreu, G. 2003. Ecología de la anidación de Crocodylus acutus (Reptilia: Crocodylidae) en la desembocadura del río Cuitzmala, Jalisco, México. Acta Zoológica Mexicana (n.s.)
89:111-128.

Casas-Andreu, G. y A. Guzmán. 1970. Estado actual de las investigaciones sobre cocodrilos mexicanos. Serie de Divulgación 3. Instituto Nacional de Investigaciones Biológicas Pesqueras, México, D.F. 50 p.

Casas-Andreu, G y B. A. Rogel. 1986. Observaciones sobre los nidos y nidadas de Crocodylus moreletii en México. Anales del Instituto de Ciencias del Mar y Limnología. Universidad Nacional Autónoma de México, Serie Zoología 13:123-303.

Casas-Andreu, G. y G. Barrios-Quiroz. 1997. Nuevos aportes a la anidación (1990-1993) de Crocodylus moreletii en cautiverio en Tabasco, México. IV Reunión Regional del Grupo de Especialistas de Cocodrilos de América Latina y el Caribe. Universidad Juárez Autónoma de Tabasco.4-7 agosto, Villahermosa, Tabasco, p. 21-25.

Casas-Andreu, G., J. F. Iracheta y H. Saracho, 1993. Anidación de Crocodylus moreletii en cautiverio en Tabasco, México. Memorias de la $1^{\text {a }}$ Reunión Regional del CSG, Grupo de Especialistas en Cocodrilos de la UICN, 11-14 de Noviembre, Santa Marta, Colombia, p.118-133.

Chabreck, R. H. 1967. Alligator farming hins. Wildlife and Fisheries Commission report, Lousiana. 21 p.

Chabreck, R. H. 1978. Collection of American alligators eggs for artificial incubation. Wildlife Society Bulletin 6:253-256.

Charnov, E. L. 1993. Life history invariants. Some exploration in symmetry in evolutionary ecology. Oxford University Press, New York.166.

Defaure, J. P. 1987. La reproducción de los cocodrilos. Mundo Científico 6:970-979.

Deitz, D. C. y T. C. Hines. 1980. Alligator nesting in North Central Florida. Copeia 1980:249-258.

De La Ossa Velásquez J. L. y A. Sampedro Marín. 2001. Densidad de manejo y alimentación adecuados para la cría en cautiverio de Crocodylus acutus (Crocodylia : Crocodylidae). Revista Biología. 2:105-110.

Del Real, V. F. 1983. Observaciones sobre la reproducción y el crecimiento de crías de Crocodylus moreletii, en cautiverio con algunas indicaciones sobre el costo de su comercialización. Tesis, Facultad de Ciencias, Universidad Nacional Autónoma de México. México D. F. 96 p.

Ferguson, M. W. J. 1981. Extrinsic microbial degradation of the alligator eggshell. Science 214:1135-1137.

Ferguson, M. W. J. 1985. Reproductive biology and embryology of the crocodilians In Biology of the Reptilia development, C. Gans, F. Billet y P. F. A. Maderson (eds.). John Wiley, New York. p. 329-491.

Foggin, M. C. 1987. Diseases and disease control in crocodile farms in Zimbawe. In Wildlife management. Crocodiles and alligators, G. J. W. Webb, S. C. Manolis and P. J. Whitehead, (eds.). Surrey Beatty, Chipping Norton, New South Wales, in association with the Conservation Commission of the Northern Territory [Australia]. p. 351-362. 
García de Miranda, E. 1981. Modificaciones al sistema de clasificación climática de Köepen. Instituto de Geografía, UNAM, México, D.F. 243.

Garrick, L. D. y J. W. Lang. 1977. Social signals and behaviors of adult alligators and crocodiles. American Zoologist 17:225239.

Greer, A. E. 1970. Evolutionary and systematic significance of crocodilian nesting habits. Nature 237:523-524.

Greer, A. E. 1975. Clutch size in crocodilians. Journal of Herpetology 9:319-322.

Hall, P. M. y D. R. Johnson. 1987. Nesting biology of Crocodylus novaeguineae in lake Murray District, Papua New Guinea. Herpetologica 43:249-258.

Honegger, R. E. 1971. Zoo breeding and crocodile banking. In Crocodile Specialist Group, IUCN Proceedings 1st Working Meetings, 6-10 September, 1965, Morges. p. 86-97.

Huerta, M. P. 1986. Etología, reproducción y biometría del cocodrilo (Crocodylus moreletii Dumeril, Bibron y Dumeril), en cautiverio. Tesis, Escuela Nacional de Ciencias Biológicas, Instituto Politécnico Nacional, México. D. F. 102 p.

Hunt, R. H. 1973. Breeding of spectacled caiman at Atlanta Zoo. International Zoo Yearbook, 13:103-105.

Hunt, R. H. 1975. Maternal behavior by adult Morelet's crocodile (Crocodylus moreletii) Copeia 1975:763-764.

Hunt, R. H. 1980. Propagation of Morelet's crocodile. In Reproductive biology and diseases of captive reptiles, J. Murphy y J. Collins (eds.) Society for the Study of Amphibians and Reptiles, Meseraull, Kansas,. p. 161-165.

Iverson, J. B., E. L Barthelmess, G. R. Smith y C. E. DeRivera. 1991. Growth and reproduction in the mud turtle Kinosternon hirtipes in Chihuahua, Mexico. Journal of Herpetology. 25:64-72.

Iverson, J. B., H. Higgins, A. Sirulnik y C. Griffiths. 1997. Local and geographic variation in the reproductive biology of the snapping turtle (Chelydra serpentina). Herpetologica 53:96117

Joanen, T. y L. McNease. 1977. Artificial incubation of alligator eggs, and posthaching culture in controlled environmental chambers. Proceeding Annales Workshop Wildlife. Mariculture Society 8: 482- 491.

Joanen, T. y L. McNease. 1987. Alligator farming research in Louisiana, USA. In Wildlife management. Crocodiles and alligators, G. J. W. Webb, S. C. Manolis y P. J. Whitehead (eds.). Surrey Beatty, association with the Conservation Commission of the Northern Territory. p. 329-340.

Joanen, T. y L. McNease. 1989. Ecology and physiology of nesting and early development of the American alligator. American Zoologist 29:987-988.

Lance, V. A. 1987. Hormonal control of reproduction in crocodilians. In Wildlife management. Crocodiles and alligators, G. J. W. Webb, S. C. Manolis y P. J. Whitehead, (eds.). Surrey Beatty, Chipping Norton, New South Wales, in association whit the Conservation Commission of the Northern
Territory [Australia]. p. 409-415.

Lang. J. W. 1987. Crocodilian behavior: implications for management. In Wildlife management crocodiles and alligators, G. J. W. Webb, S. C. Manolis y P. J. Whitehead (eds.). Surrey Beatty, Chipping Norton, New South Wales, in association whit the Conservation Commission of the Northern Territory [Australia]. p. 273-294

Larriera, A., C. Piña, P. Siroski y L. M. Verdade. 2004. Allometry of reproduction in wild broad-snouted caimans Caiman latirostris. Journal of. Herpetology 38:301-304.

Martín de Lucenay, A.1942. Nuestra riqueza pesquera, el cocodrilo mexicano. Revista General de Marina, Secretaría de Marina, México, D.F. p. 24-27.

Pérez-Higadera, G. 1980. Notes on nesting of Crocodylus moreletii in Southern Veracruz, México. Bulletin of the Maryland Herpetological Society 16:52-53.

Pérez-Higareda, G., A. Rogel y H. M. Smith. 1989. The courtship and mating Behavior of Morelet's crocodile (Crocodylus moreletii) in southern Veracruz, Mexico. Bulletin of the Chicago Herpetological Society 7:131-132.

Platt, S.G. 1996. Ecology and status of Morelet's crocodile in Belize. PhD dissertation, Clemson University, South Carolina. $187 \mathrm{p}$.

Platt, S. G., T. R. Rainwater, J. B. Thorbjarnarson y S. T. McMurry. 2008. Reproductive dynamics of a tropical freshwater crocodilian: Morelet's crocodile in northern Belize. Journal of Zoology 275:177-189.

Pooley, A. C. 1971. Crocodile rearing and restocking. In Crocodiles, IUCN Publications, New Series. Supplementary Paper 32. International Union for Conservation of Nature and Natural Resources Gland.p. 104-130.

Pooley, A. C. 1990. Bases para la crianza de cocodrilos en zonas remotas. In Crocodiles. Proceedings of the 10th Working Meeting of the Specialist Group, IUCN, 23-27 de abril. The World Conservation Union, Gland. Switzerland. p. 345.

Staton, M. A. y J. R. Dixon. 1977. Breeding biology of the spectacled caiman, Caiman crocodilus crocodilus in the Venezuelan Llanos. U. S. Department of Interior, Fish and Wildlife Service, Wildlife Research Report 5:1-21.

Stearns, S. C. 1992. The evolution of life histories. Oxford University Press, New York. p 249.

Thorbjarnarson, J. 1989. Ecology of the American crocodile (Crocodylus acutus). In Crocodiles: their ecology, management, and conservation. P.M. Hall, (eds.). Gland, Switzerland: IUCN - The World Conservation Union, Gland. p. 228-258.

Thorbjarnarson, J. B. 1996. Reproductive characteristics of the order Crocodilia. Herpetologica 52:8-24.

Thorbjarnarson, J. B. y G. Hernández 1993. Reproductive ecology of the Orinoco crocodile (Crocodylus intermedius) in Venezuela I: nesting ecology and eggs and clutch relationships. Journal of Herpetology 27:363-370.

Verdade L. M. 2001. Allometry of reproduction in broad-snouted 
caiman (Caiman latirostris). Brazilian Journal of Biology 61:431-435.

Webb, G. J.W. y H. Cooper-Preston 1989. Effects of temperature on crocodiles and evolution of reptilian oviparity. American Zoologist. 29: 935-971.

Webb, G. J. W., H. Messel y W. Magnusson. 1977. The nesting of Crocodylus porosus in Arnhem Land, northern Australia. Copeia 1977:238-249.

Yadav, R. M. 1969. Breeding the mugger crocodile at Jairpur Zoo. International ZooYearbook. 9:3.

Youngprapakorn, U. 1972. The breeding of crocodiles in captivity at Samut Prakan, Thailand. IUCN Publications Support 32:98-101. 
